Published in final edited form as:

J Surg Res. 2008 November ; 150(1): 49-52. doi:10.1016/j.jss.2007.09.020.

\title{
Is Hashimoto's thyroiditis a risk factor for papillary thyroid cancer?
}

\author{
Daniel Repplinger, B.S., Anna Bargren, B.S, Yi-Wei Zhang, B.S., Joel Adler, B.A., Megan \\ Haymart, M.D., and Herbert Chen, M.D., F.A.C.S. \\ Section of Endocrine Surgery, Department of Surgery, University of Wisconsin, Madison, WI
}

\section{Abstract}

Background-Hashimoto's thyroiditis is the most common cause of hypothyroidism and is characterized by gradual autoimmune mediated thyroid failure with occasional goiter development. Hashimoto's (HT) is seven times more likely to occur in women than in men. Papillary thyroid cancer (PTC), the most prevalent form of cancer in the thyroid, is 2.5 times more likely to develop in women than men. Given the relatively high prevalence of these diseases and the increased occurrence in women, we analyzed data from our institution to determine if there is a correlation between Hashimoto's thyroiditis and PTC in women.

Methods-From May 1994 to January 2007, 1198 patients underwent thyroid surgery at our institution. Of these, 217 patients were diagnosed with HT (196 women, 21 men). The data from these patients were statistically analyzed using SPSS.

Results-PTC occurred in 63 of 217 (29\%) HT patients and 230 of 981 (23\%) patients without HT $(\mathrm{p}=0.051)$. Of these groups, $41(65 \%)$ and $158(69 \%)$ patients, respectively, had tumor sizes of $\geq 1.0 \mathrm{~cm}$. 56/196 women (29\%) with HT had coexistent PTC compared to 160/730 women (22\%) without HT ( $\mathrm{p}=0.03)$. Among women with any type of thyroid malignancy, $56 / 59$ cases $(95 \%)$ with HT had PTC compared to $159 / 196$ cases $(81 \%)$ in women without HT ( $\mathrm{p}=0.006)$. Additionally, female HT patients with goiters had a significantly lower rate of PTC ( $9 \%$ vs. $36 \%$, p $<0.001$ ) when compared to women without goiters. These differences were not observed in men with HT.

Conclusions-These data demonstrate that HT is associated with an increased risk of developing PTC. Female patients with HT undergoing thyroidectomy are 30\% more likely to have PTC. Thus, more aggressive surveillance for PTC may be indicated in patients with HT, especially in women.

\section{Keywords}

Hashimoto's thyroiditis; papillary thyroid carcinoma

\section{Introduction}

Hashimoto's thyroiditis is the most common inflammatory thyroid disease as well as the most common cause of hypothyroidism in the United States as it affects 22 per 100,000 individuals. [1-3] It is characterized by gradual autoimmune-mediated thyroid failure with occasional

\footnotetext{
Corresponding Author: Herbert Chen, M.D., F.A.C.S., H4/750 Clinical Science Center, 600 Highland Avenue, Madison, WI 53792 , Phone: (608) 263-1387, Fax: (608) 263-7652, E-mail: chen@ surgery.wisc.edu.

Publisher's Disclaimer: This is a PDF file of an unedited manuscript that has been accepted for publication. As a service to our customers we are providing this early version of the manuscript. The manuscript will undergo copyediting, typesetting, and review of the resulting proof before it is published in its final citable form. Please note that during the production process errors may be discovered which could affect the content, and all legal disclaimers that apply to the journal pertain.
} 
goiter development. The disease occurs more frequently in females, with published gender prevalence ratios ranging from 5 to $20: 1$. [1,3,4]

Similar to Hashimoto's thyroiditis, papillary thyroid cancer (PTC) is a relatively common disease. It is the most prevalent manifestation of thyroid cancer, representing $70-80 \%$ of all diagnosed thyroid cancers. [5,6] It occurs more frequently in women with prevalence ratios ranging from 2.5 to $4.0: 1$. [4]

The relationship between Hashimoto's thyroiditis and papillary thyroid carcinoma was first proposed by Dailey, et al. in 1955. [7] Since this initial description, the association between the two diseases has been highly debated in the literature and the relationship remains controversial. Studies to-date establish 11-36\% of patients with coexistent Hashimoto's thyroiditis/PTC disease. [2,8-11] Okayasu, et al. determined a clear association between the two diseases among patients of differing ethnic origin. [12] Due to the ongoing debate, as well as the high prevalence of both diseases, this study was undertaken to determine the association between Hashimoto's thyroiditis and PTC.

\section{Materials and Methods}

From May 1994 to January 2007, 1198 patients underwent thyroid surgery at the University of Wisconsin. Of these patients, 217 (196 female, 21 male) were diagnosed with Hashimoto's thyroiditis, which was confirmed by permanent section. For all patients, data were collected by retrospective chart review for patient demographics, gland weight, nodule size, presence of a goiter, and malignancy.

Data analysis was conducted using statistical software (SPSS Graduate Pack 10.0, SPSS Inc., Chicago, IL.). In analysis of these data, $\mathrm{X}^{2}$, Fisher's exact test, and one-way analysis of variance (ANOVA) was utilized when appropriate. With regard to the results, statistical significance was defined as $p \leq 0.05$. The collection of patient data and subsequent analysis was approved by the University of Wisconsin Human Subjects Institutional Review Board.

\section{Results}

\section{Patient characteristics}

Of the 1198 patients who underwent thyroid surgery over a 13 year time-span, 18\% (217) were diagnosed with Hashimoto's thyroiditis based on final pathology. When comparing patients with Hashimoto's thyroiditis to those without Hashimoto's thyroiditis, there was no significant difference between demographic variables (Table 1) with the exception of females being more likely to have Hashimoto's thyroiditis and smaller nodule size seen in Hashimoto's thyroiditis patients. $90 \%$ of patients with Hashimoto's thyroiditis were female while only $74 \%$ of patients without Hashimoto's thyroiditis were female $(p<0.001)$. The nodule sizes between Hashimoto's thyroiditis patients and patients without Hashimoto's thyroiditis differed significantly at $2.34 \pm 0.14 \mathrm{~cm}$ and $2.71 \pm 0.07 \mathrm{~cm}$, respectively $(p=0.045)$. Although patients with Hashimoto's thyroiditis appeared to be slightly younger than those without Hashimoto's thyroiditis (mean age $46 \pm 1$ vs. $49 \pm 1$ ), this difference was not significant ( $p=0.076$ ). Of note, there was a trend towards smaller gland size in patients with Hashimoto's thyroiditis. The average gland weight of Hashimoto's thyroiditis patients was $30.3 \pm 2.5$ grams and the average for patients without Hashimoto's thyroiditis was $39.8 \pm 2.2$ grams $(p=0.053)$. In addition, patients with Hashimoto's thyroiditis exhibited a higher rate of PTC overall compared to patients without Hashimoto's thyroiditis ( $29 \%$ vs. $23 \%, p=0.051) .33 \%$ of males with Hashimoto's thyroiditis also had PTC whereas $28 \%$ of males without Hashimoto's thyroiditis had PTC ( $p=0.379)$. Female patients with Hashimoto's thyroiditis showed a more dramatic 
difference in the incidence of PTC contrasted to females without Hashimoto's thyroiditis (29\% vs. $22 \%, p=0.033)$.

\section{Hashimoto's thyroiditis patients}

Within the subgroup of patients with Hashimoto's thyroiditis, there was no significant difference between pathologic variables such as age, gland weight, and nodule size (Table 2). Among patients with Hashimoto's thyroiditis, 63 (29\%) had concurrent PTC, while 154 (71\%) did not have PTC. However, the presence of a goiter was inversely associated with risk of malignancy in those patients with Hashimoto's thyroiditis. Concerning all Hashimoto's thyroiditis patients, 63 (29\%) also had a goiter. Of Hashimoto's thyroiditis patients without PTC, 56/154 (36\%) had a goiter, while only 7/63 (11\%) of patients with PTC had a goiter $(p<0.001)$. Only 5/56 (9\%) female Hashimoto's thyroiditis patients with PTC had a goiter, while 51/140 (36\%) female Hashimoto's thyroiditis patients without PTC had a goiter $(p<0.001)$.

\section{Type of malignancy}

In the subgroup of patients with Hashimoto's thyroiditis, the vast majority of all malignancies were PTC (63/67, 94\%). Although PTC was also the most common malignancy in patients without Hashimoto's thyroiditis, it was significantly less common relative to patients with Hashimoto's thyroiditis $(229 / 298,76 \%, p=0.001)$. There was a significantly greater percentage of PTC occurrence in females with Hashimoto's thyroiditis compared to females without Hashimoto's thyroiditis $(p=0.006)$. This trend was not significantly reproducible in male patients (Table 3).

\section{Discussion}

Since first being described by Dailey, et al. in 1955, the association between Hashimoto's thyroiditis and PTC has been widely disputed and remains so in the literature. [2,7-11] Given the relatively high incidence of both of these diseases, we further investigated this relationship. At our institution, of the 217 patients with Hashimoto's thyroiditis, 63 had coexistent PTC. If subdivided by gender, this was statistically significant for women with Hashimoto's thyroiditis. $29 \%$ of female Hashimoto's thyroiditis patients had concomitant PTC. When compared to other females without Hashimoto's thyroiditis, women with Hashimoto's thyroiditis were found to be $30 \%$ more likely to have coexisting PTC. The findings in this patient population support the previous studies linking Hashimoto's thyroiditis and PTC. [2,8-11] The data from male patients did not yield statistically significant results. While 33\% of male Hashimoto's thyroiditis patients had coexistent PTC, $28 \%$ of male patients without Hashimoto's thyroiditis also had PTC. The lack of significance is most likely due to a small sampling size. Although the $p$-value did not meet our criteria for statistical significance, the data would possibly change with additional patients to analyze.

This study also found that the presence of a goiter is associated with a lower rate of PTC in Hashimoto's thyroiditis patients (11\% vs. $36 \%$ ). However, since all the patients analyzed in this study underwent thyroid surgery, the selection of these patients must be noted. It is possible that many of the patients with a goiter underwent surgery due to compressive symptoms rather than nodule biopsy that warranted gland resection. That said, the high statistical significance of this result still merits investigation. In 2004, Gasbarri, et al. determined that the diagnosis of Hashimoto's thyroiditis actually represents a variety of disease mechanisms which influence the clinical presentation of the disease. [13] Therefore, one may speculate that a particular mechanism may predispose a patient to varying thyroid growth, including goiters or carcinomas. 
Since the causative relationship between Hashimoto's thyroiditis and PTC is not yet clear, careful observation of Hashimoto's thyroiditis patients is recommended. There have been a number of proposed mechanisms of both of these diseases in the literature, along with some attempts to explain the association. For example, Wirtschafter, et al. described expression of the RET/PTC1 and RET/PTC3 oncogenes in Hashimoto's patients. [14] Arif, et al. also supported this hypothesis, demonstrating both diseases have similar immunohistochemical staining, morphological features and molecular profile in regards to the RET/PTC gene rearrangement. [15] In addition, Unger, et al. found expression of p63 in Hashimoto's patients with papillary thyroid cancer. [16] This was further examined by Burstein, et al. who proposed the two diseases are both initiated by pluripotent p63-positive stem cell remnants. [17]

In this study, there was a trend in Hashimoto's thyroiditis patients for the coexistence of PTC; a finding that becomes statistically significant in female Hashimoto's thyroiditis patients. Women with Hashimoto's thyroiditis have a 30\% increased risk of having PTC compared to women without Hashimoto's thyroiditis. In addition, among all Hashimoto's thyroiditis patients, an overwhelming majority of malignancies were papillary carcinomas. Thus, it is plausible to assume that Hashimoto's thyroiditis and PTC may be associated diseases. From the data presented above, it can be concluded that a heightened suspicion of PTC may be warranted in patients with Hashimoto's thyroiditis, especially females. We recommend these patients receive periodic thyroid evaluations to assess any nodules present. If there is a nodule greater than one centimeter, ultrasound-guided FNA is advised.

\section{References}

1. Lal, G.; Clark, OH. Textbook of endocrine surgery. Philadelphia: Saunders; 2005. Chronic Thyroiditis; p. 38-40.

2. Singh B, Shaha AR, Trivedi H, Carew JF, Poluri A, Shah JP. Coexistent Hashimoto's thyroiditis with papillary thyroid carcinoma: impact on presentation, management, and outcome. Surgery 1999;126:1070-1076. [PubMed: 10598190]discussion 1076-7

3. Kumar, V.; Robbins, SL. Robbins basic pathology. Philadelphia, PA: Saunders/Elsevier; 2007. The endocrine system; p. 731-732.p. 735-736.

4. Bloodworth, JMB.; Lechago, J.; Gould, VE. Bloodworth's endocrine pathology. Baltimore, Md: Williams \& Wilkins; 1997. The thyroid; p. 178-181.p. 197-206.

5. Cheema Y, Olson S, Elson D, Chen H. What is the biology and optimal treatment for papillary microcarcinoma of the thyroid? J. Surg. Res 2006;134:160-162. [PubMed: 16780882]

6. Cheema Y, Repplinger D, Elson D, Chen H. Is tumor size the best predictor of outcome for papillary thyroid cancer? Ann. Surg. Oncol 2006;13:1524-1528. [PubMed: 17006742]

7. Dailey ME, Lindsay S, Skahen R. Relation of thyroid neoplasms to Hashimoto disease of the thyroid gland. AMA Arch. Surg 1955;70:291-297. [PubMed: 13227748]

8. Boi F, Lai ML, Marziani B, Minerba L, Faa G, Mariotti S. High prevalence of suspicious cytology in thyroid nodules associated with positive thyroid autoantibodies. Eur. J. Endocrinol 2005;153:637642. [PubMed: 16260421]

9. Cipolla C, Sandonato L, Graceffa G, Fricano S, Torcivia A, Vieni S, Latteri S, Latteri MA. Hashimoto thyroiditis coexistent with papillary thyroid carcinoma. Am. Surg 2005;71:874-878. [PubMed: 16468540]

10. Liu LH, Bakhos R, Wojcik EM. Concomitant papillary thyroid carcinoma and Hashimoto' thyroiditis. Semin. Diagn. Pathol 2001;18:99-103. [PubMed: 11403259]

11. Pino Rivero V, Guerra Camacho M, Marcos Garcia M, Trinidad Ruiz G, Pardo Romero G, Gonzalez Palomino A, Blasco Huelva A. The incidence of thyroid carcinoma in Hashimoto's thyroiditis. Our experience and literature review. An Otorrinolaringol. Ibero. Am 2004;31:223-230. [PubMed: 15259844]

12. Okayasu I, Fujiwara M, Hara Y, Tanaka Y, Rose NR. Association of chronic lymphocytic thyroiditis and thyroid papillary carcinoma. A study of surgical cases among Japanese, and white and African Americans. Cancer 1995;76:2312-2318. [PubMed: 8635037] 
13. Gasbarri A, Sciacchitano S, Marasco A, Papotti M, Di Napoli A, Marzullo A, Yushkov P, Ruco L, Bartolazzi A. Detection and molecular characterisation of thyroid cancer precursor lesions in a specific subset of Hashimoto's thyroiditis. Br. J. Cancer 2004;91:1096-1104. [PubMed: 15292926]

14. Wirtschafter A, Schmidt R, Rosen D, Kundu N, Santoro M, Fusco A, Multhaupt H, Atkins JP, Rosen MR, Keane WM, Rothstein JL. Expression of the RET/PTC fusion gene as a marker for papillary carcinoma in Hashimoto's thyroiditis. Laryngoscope 1997;107:95-100. [PubMed: 9001272]

15. Arif S, Blanes A, Diaz-Cano SJ. Hashimoto's thyroiditis shares features with early papillary thyroid carcinoma. Histopathology 2002;41:357-362. [PubMed: 12383219]

16. Unger P, Ewart M, Wang BY, Gan L, Kohtz DS, Burstein DE. Expression of p63 in papillary thyroid carcinoma and in Hashimoto's thyroiditis: a pathobiologic link? Hum. Pathol 2003;34:764-769. [PubMed: 14506636]

17. Burstein DE, Nagi C, Wang BY, Unger P. Immunohistochemical detection of p53 homolog p63 in solid cell nests, papillary thyroid carcinoma, and hashimoto's thyroiditis: A stem cell hypothesis of papillary carcinoma oncogenesis. Hum. Pathol 2004;35:465-473. [PubMed: 15116328] 
Table 1

Patient Characteristics*

\begin{tabular}{|c|c|c|c|}
\hline & Hashimoto's & No Hashimoto's & $p$ value ${ }^{* *}$ \\
\hline $\mathbf{N}$ & 217 & 981 & \\
\hline Age (years) & $46 \pm 1$ & $49 \pm 1$ & 0.076 \\
\hline \multicolumn{4}{|l|}{ Gender } \\
\hline Male & $10 \%$ & $26 \%$ & $<0.001$ \\
\hline Female & $90 \%$ & $74 \%$ & \\
\hline Gland Weight (g) & $30.3 \pm 2.5$ & $39.8 \pm 2.2$ & 0.053 \\
\hline Nodule Size $(\mathrm{cm})$ & $2.34 \pm 0.14$ & $2.71 \pm 0.07$ & 0.045 \\
\hline \multicolumn{4}{|c|}{ Percent with Papillary Thyroid } \\
\hline \multicolumn{4}{|c|}{ Cancer } \\
\hline All Patients & $29 \%$ & $23 \%$ & 0.051 \\
\hline Male & $33 \%$ & $28 \%$ & 0.379 \\
\hline Female & $29 \%$ & $22 \%$ & $\mathbf{0 . 0 3 3}$ \\
\hline
\end{tabular}

Values reported as mean \pm SEM

***isher's exact test 
Table 2

Hashimoto'S Patients *

\begin{tabular}{|c|c|c|c|}
\hline & Papillary Thyroid Cancer & $\begin{array}{c}\text { No Papillary Thyroid } \\
\text { Cancer }\end{array}$ & $p$ value $* *$ \\
\hline $\mathbf{N}$ & 63 & 154 & \\
\hline Age (years) & $44 \pm 2$ & $47 \pm 1$ & 0.339 \\
\hline Gland Weight (g) & $24.1 \pm 3.0$ & $32.8 \pm 3.3$ & 0.122 \\
\hline Nodule Size $(\mathrm{cm})$ & $2.38 \pm 0.35$ & $2.34 \pm 0.16$ & 0.912 \\
\hline \multicolumn{4}{|l|}{ Goiters } \\
\hline All Patients & $11 \%$ & $36 \%$ & $<0.001$ \\
\hline Male & $29 \%$ & $36 \%$ & 0.572 \\
\hline Female & $9 \%$ & $36 \%$ & $<0.001$ \\
\hline
\end{tabular}

\footnotetext{
Values reported as mean \pm SEM

** Fisher's exact test
} 
Table 3

Type of Malignancy

\begin{tabular}{lccc}
\hline & Hashimoto's & No Hashimoto's & $p$ value * \\
\hline $\mathbf{N}$ & 67 & 298 & \\
Percent with Papillary Thyroid & & & \\
Cancer & $63 / 67(94 \%)$ & $229 / 298(76 \%)$ & $\mathbf{0 . 0 0 1}$ \\
$\quad$ All Patients & $7 / 8(88 \%)$ & $70 / 102(69 \%)$ & 0.245 \\
Male & $56 / 59(95 \%)$ & $159 / 196(81 \%)$ & $\mathbf{0 . 0 0 6}$ \\
Female & & & \\
\hline
\end{tabular}

Fisher's exact test 